\title{
Vortex Lattice Structural Transitions: a Ginzburg-Landau Model Approach
}

\author{
A. D. Klironomos and Alan T. Dorsey \\ Department of Physics, University of Florida, P.O. Box 118440, Gainesville, Florida 32611-8440
}

(Dated: November 23, 2018)

\begin{abstract}
We analyze the rhombic to square vortex lattice phase transition in anisotropic superconductors using a variant of Ginzburg-Landau (GL) theory. The mean-field phase diagram is determined to second order in the anisotropy parameter, and shows a reorientation transition of the square vortex lattice with respect to the crystal lattice. We then derive the long-wavelength elastic moduli of the lattices, and use them to show that thermal fluctuations produce a reentrant rhombic to square lattice transition line, similar to recent studies which used a nonlocal London model.

PACS numbers: 74.25.Qt,74.40.+k,74.70.Dd
\end{abstract}

Vortex lattices in superconductors with four-fold anisotropy can exhibit a rhombic to square structural phase transition due to a competition between the anisotropy (favoring a square lattice) and the repulsive vortex-vortex interaction (favoring a triangular lattice). This transition has been observed in the borocarbide family of superconductors $R \mathrm{Ni}_{2} \mathrm{~B}_{2} \mathrm{C}$ (with $R$ a rare earth element), whose anisotropy is due to lattice effects [1, 2, 3, 4, 5], and more recently in the overdoped cuprate superconductor $\mathrm{La}_{1.83} \mathrm{Sr}_{0.17} \mathrm{CuO}_{4+\delta}$ [6], whose anisotropy is due to unconventional superconducting order. Recent small angle neutron scattering (SANS) data for $\mathrm{LuNi}_{2} \mathrm{~B}_{2} \mathrm{C}$ [1] show the transition line in the $H-T$ plane curving upward and avoiding $H_{c 2}$, contrasting with mean-field predictions and underscoring the importance of thermal fluctuations even in this low temperature superconductor. Some features of the data can be understood using a nonlocal London model developed by Kogan and collaborators [7, 8]; however, the London model is ostensibly only applicable at low magnetic inductions, leaving as an open question the behavior close to $H_{c 2}$. An alternate explanation for the reentrant behavior of the vortex lattice transition is also available in the literature 9 .

In this Letter we study the effect of thermal fluctuations on the rhombic to square vortex lattice transition using an anisotropic GL model. We first determine the mean-field phase diagram to second order in the anisotropy parameter, and find that even at this level GL theory predicts a novel reorientation transition of the square vortex lattice with respect to the crystal lattice, an effect overlooked in previous GL studies. We then derive the (nondispersive) elastic moduli of the lattices using methods first pioneered by Brandt [10] for isotropic superconductors. Finally, these moduli are used to determine the fluctuation renormalized phase boundary in the $H-T$ plane. For parameters typical of the borocarbides we find a reentrant phase boundary, in agreement with the nonlocal London model results of Ref. 11]. As GL theory is expected to be valid near $H_{c 2}$, our results nicely complement and extend the nonlocal London model results.

Accounting for anisotropy, the linearized first GL equa- tion (in dimensionless units) is

$$
\Pi^{2} \psi-\psi-\epsilon\left(\Pi_{x}^{2}-\Pi_{y}^{2}\right)^{2} \psi=0,
$$

where $\boldsymbol{\Pi}=(\boldsymbol{\nabla} / i \kappa-\mathbf{A})$ is the covariant derivative and $\epsilon$ is the dimensionless anisotropy parameter. For the cuprates the anisotropic term can be derived rigorously from the two field $s$ and $d$-wave GL free energy [12, 13. by integrating out the $s$-wave term [14]. For the conventional, but highly anisotropic, borocarbides it is a phenomenological term that will be sufficient to capture all the interesting physics. The symmetry breaking properties of the anisotropic term dictate the introduction of the orientation angle $\phi$ of the vortex lattice relative to the underlying crystal lattice - this term provides an effective coupling of the vortex and crystal lattices, and makes structural transitions possible.

We have generalized the virial theorem of superconductivity [15, 16] to include the anisotropic term in the extended GL theory [17], with the result

$$
\begin{aligned}
\frac{1}{4 \pi} \mathbf{H} \cdot \mathbf{B}=\left\langle|\psi|^{2}-|\psi|^{4}+\frac{\mathbf{B}^{2}(\mathbf{x})}{4 \pi}\right. & \left.-2 \epsilon \operatorname{Re}\left[\psi^{*}\left(\Pi_{x}^{2}-\Pi_{y}^{2}\right)^{2} \psi\right]\right\rangle,
\end{aligned}
$$

where the brackets \langle\rangle denote spatial averaging.

Using the generalizations of the Abrikosov identities derived with the help of Eq. (2), the free energy can be cast into the compact form

$$
F=B^{2}-\frac{(\kappa-B)^{2}}{\left(2 \kappa^{2}-1\right) \beta_{A}+1}\left\{1-\frac{\Gamma}{\left(2 \kappa^{2}-1\right) \beta_{A}+1}\right\},
$$

where $\beta_{A}$ is the usual Abrikosov parameter and $\Gamma$ is the correction to the isotropic result,

$$
\begin{aligned}
\Gamma=4 \epsilon\{ & \frac{\operatorname{Re}\left\langle|\psi|^{2} \psi^{*}\left(\Pi_{x}^{2}-\Pi_{y}^{2}\right)^{2} \psi\right\rangle}{\left\langle|\psi|^{2}\right\rangle^{2}} \\
& \left.+\beta_{A}\left(2 \kappa^{2}-1\right) \frac{\operatorname{Re}\left\langle\psi^{*}\left(\Pi_{x}^{2}-\Pi_{y}^{2}\right)^{2} \psi\right\rangle}{\left\langle|\psi|^{2}\right\rangle}\right\} .
\end{aligned}
$$

Throughout this paper we will use the convenient parameterization $\rho+i \sigma=(\bar{b} / \bar{a}) \exp (i \theta)$, where $\bar{b}$ and $\bar{a}$ are the 
magnitudes of the two basis vectors of the vortex lattice and $\theta$ is the apex angle.

Equation (11) was solved perturbatively to second order in the anisotropy parameter $\epsilon$, extending previous first order results [18, 19, 20]. The second order calculation is necessary to justify the use of perturbation theory and at the same time to quantify the accuracy of the first order correction. More importantly, we uncover new and unexpected physics at second order: a reorientation transition of the square vortex lattice with respect to the crystal lattice. The quantities $\beta_{A}$ and $\Gamma$ are given in terms of rapidly converging sums involving $\rho, \sigma$, and $\phi$,

$$
\begin{gathered}
\beta_{A}=\beta_{0}+(\epsilon b) \beta_{1}+(\epsilon b)^{2}\left[\gamma_{1}+\gamma_{2}+5 \beta_{1}-\frac{3}{4} \beta_{0}\right] \\
\Gamma=8 \epsilon b^{2}\left\{\beta_{A}\left(2 \kappa^{2}-1\right)[1+3(\epsilon b)]+\beta_{0}\left[1+\frac{3}{2}(\epsilon b)\right]\right. \\
\left.+\beta_{1}[1+6(\epsilon b)]+2(\epsilon b)\left[\gamma_{1}+\gamma_{2}\right]\right\}
\end{gathered}
$$

where the functions $\beta_{0}, \beta_{1}, \gamma_{1}, \gamma_{2}$ are periodic in $\rho$ with period 1 , and are given by

$$
\begin{gathered}
\beta_{0}=\sum_{n m}^{\prime} A_{n m} \\
\beta_{1}=\operatorname{Re}\left\{e^{4 i \phi} \sum_{n m}^{\prime} A_{n m}\left[8 \pi^{2} \sigma^{2} n^{4}-6 \pi \sigma n^{2}+\frac{3}{8}\right]\right\} \\
\gamma_{1}=\operatorname{Re}\left\{e ^ { 8 i \phi } \sum _ { n m } ^ { \prime } A _ { n m } \left[16 \pi^{4} \sigma^{4} n^{8}-12 \pi^{3} \sigma^{3} n^{6}\right.\right. \\
\left.\left.\quad+\frac{3}{4} \pi^{2} \sigma^{2} n^{4}-\frac{45}{16} \pi \sigma n^{2}+\frac{105}{256}\right]\right\} \\
\gamma_{2}=\sum_{n m}^{\prime} A_{n m}\left[16 \pi^{4} \sigma^{4} n^{4} m^{4}-12 \pi^{3} \sigma^{3} n^{2} m^{2}\left(n^{2}+m^{2}\right)\right. \\
+\frac{3}{4} \pi^{2} \sigma^{2}\left(n^{4}+m^{4}+36 n^{2} m^{2}\right) \\
\left.-\frac{45}{16} \pi \sigma\left(n^{2}+m^{2}\right)+\frac{105}{256}\right]
\end{gathered}
$$

where $A_{n m}=\sqrt{\sigma} e^{2 i \pi \rho\left(n^{2}-m^{2}\right)} e^{-2 \pi \sigma\left(n^{2}+m^{2}\right)}$. The prime denotes a double sum: one over $n, m$ and a second with $n$ and $m$ replaced by $(n+1 / 2)$ and $(m+1 / 2)$ respectively. We perform a numerical minimization of the free energy (3) with respect to $\rho, \sigma$, and $\phi$ to obtain the mean-field vortex lattice structure as a function of the field and temperature. We find a continuous transition from a rhombic to square phase as $\epsilon$ is increased. The second order correction only shifts the transition point by about $1 \%$ relative to the first order calculation, contrary to previous claims [21] which neglected the lifting of the orientation degeneracy by the anisotropic term. Our numerical investigations show unambiguously that a consistent exploration of the relevant phase space and an accurate determination of the global minima of the GL free energy (3) demands the inclusion of the the orientational degree of freedom $\phi$. After the structural phase transition occurs the square vortex lattice is oriented along the [110] direction, and remains in this preferred orientation until a field $b_{r}$ is reached. At that point another continuous phase transition takes place, with the vortex lattice changing its orientation while retaining its square shape. At a given $b>b_{r}$, two equivalent configurations exist: one with orientation angle $\phi>\pi / 4$, and one with $\phi^{\prime}=\pi / 2-\phi$. This reorientation transition occurs only at second order in the anisotropy $\epsilon$.

Having determined the mean-field phase diagram of the system, we now turn to the elastic properties of the lattices. Assuming that the displacement field $\mathbf{u}$ is small compared to typical lattice spacings, we calculate the elastic moduli from the GL free energy (3) in the incompressible limit, $\boldsymbol{\nabla} \cdot \mathbf{u}=0$. For simplicity we have focused on straight and parallel vortices; we do not expect the structural transition to significantly affect the tilt modulus $c_{44}$. The relevant moduli are essentially nondispersive [10], allowing us to consider uniform deformations. Now there are many ways to choose the elastic moduli from the independent components of the elastic tensor, and we will follow the lead of Miranović and Kogan [22] and use as the four independent elastic moduli for a rhombic lattice the quantities $c_{s q}, c_{66 x}, c_{66 y}$, and $c_{r}$, corresponding to four particular deformations. The socalled "squash" deformation is responsible for the transformation of the rhombic to a square vortex lattice, and the corresponding squash modulus $c_{s q}$ vanishes at the transition point. The other relevant deformations are the two pure shears in $x$ and $y$ directions (which determine $\left.c_{66 x}, c_{66 y}\right)$ and the rotation of the lattice about the direction of the magnetic field (which determines $c_{r}$ ).

All elastic moduli are obtained from the second derivative of the GL free energy (3). The elastic energy can be written as

$$
E=\left.\frac{1}{2} \mu^{2} c(\mu)\right|_{\mu \rightarrow 0}=\left.\frac{1}{2} \mu^{2} \partial_{\mu}^{2} F\right|_{\mu \rightarrow 0},
$$

where $c(\mu)$ is the elastic modulus for each particular deformation. Each elastic modulus is obtained from

$$
\begin{aligned}
c & =C_{\rho \rho} \rho_{\mu}^{2}+C_{\sigma \sigma} \sigma_{\mu}^{2}+C_{\phi \phi} \phi_{\mu}^{2} \\
& +2 C_{\rho \sigma} \rho_{\mu} \sigma_{\mu}+2 C_{\rho \phi} \rho_{\mu} \phi_{\mu}+2 C_{\sigma \phi} \sigma_{\mu} \phi_{\mu},
\end{aligned}
$$

where $C_{i j}$ are the terms originating from the derivatives of the GL free energy (3)

$$
\begin{aligned}
C_{i j}=(2 & \left.-\delta_{i j}\right)\left\{\Phi\left(b^{2}-F\right) \beta_{A, i j}+\Theta\left[\Gamma_{, i j}-\Phi\left(\beta_{A, i} \Gamma_{, j}\right.\right.\right. \\
& \left.\left.\left.+\beta_{A, j} \Gamma_{, i}+\Gamma \beta_{A, i j}-2 \Phi \Gamma \beta_{A, i} \beta_{A, j}\right)\right]\right\} . \quad
\end{aligned}
$$

The derivatives in the right hand side of Eq. (13) are with respect to $i, j=\rho, \sigma, \phi, \Phi=\left(2 \kappa^{2}-1\right) /\left[\left(2 \kappa^{2}-1\right) \beta_{A}+1\right]$, and $\Theta=(1-b)^{2} /\left[\left(2 \kappa^{2}-1\right) \beta_{A}+1\right]^{2}$. 
The calculated elastic moduli for $\epsilon=0.11$ and $\kappa=10$ (appropriate for the borocarbides) are shown in Fig. 1] The transition from a rhombic to a square vortex lattice is signaled by the vanishing of the squash modulus. At the same point the two shear moduli merge into one because of the higher symmetry of the square phase. At a still higher field we observe the reorientation transition, which occurs when $c_{r}=0$. This instability of the vortex lattice with respect to rotations also exists in the nonlocal London model 22]. There are experimental indications for this instability, although its investigation has been hampered so far by the high fields required for its observation [23]. This reorientation transition is sensitive to the value of $\kappa$, and from our results we predict that this transition will not occur in the cuprates $(\kappa \sim 100)$, as the transition point where $c_{r}=0$ moves above $H_{c 2}$.

We also note that anisotropic elasticity with a softer shear modulus for shearing along the sides of the square lattice than along the diagonals emerges naturally in this model. This response of the vortex lattice is measured by the ratio $c_{66 x} / c_{66}, \frac{\pi}{4}$, which has a maximum at the transition as can be seen in Fig. 2 It has been suggested 23] that this behavior explains the anisotropic orientational long range order observed in decoration patterns in $\mathrm{LuNi}_{2} \mathrm{~B}_{2} \mathrm{C}$, which manifests itself as a significant difference in the correlation lengths along the [110] and [110] directions.

We next turn to the effects of thermal fluctuations on the vortex lattice structure. Following Gurevich and Kogan [1], we will make the simplifying assumption that harmonic fluctuations renormalize the three variables $\rho$, $\sigma$, and $\phi$, which characterize the structure of the vortex lattice, with the result that

$$
\begin{aligned}
& \bar{\rho}=\rho\left[1-\sigma(2-\rho) \overline{u^{2}}\right] \\
& \bar{\sigma}=\sigma\left[1-\sigma(2-\rho) \overline{u^{2}}\right] \\
& \bar{\phi}=\phi+\sigma \cot \phi \overline{u^{2}}
\end{aligned}
$$

where overbars denote thermal averages. The rhombic to square transition is then determined as the locus of points in the $H-T$ plane where $c_{s q}=0$. For that purpose, the "squash" elastic modulus $c_{s q}$ is recalculated from Eq. (12) using the renormalized variables $\bar{\rho}, \bar{\sigma}, \bar{\phi}$. This is not performed self-consistently, as we do not take into account the effects of the fluctuations on the elastic moduli themselves. This approach is justified due to the fact that the mean squared displacement of the vortex $\overline{u^{2}}$ is finite at $H_{\square}(T)[11]$. There are two competing parameters, the strength of the thermal fluctuations $\chi$ and the anisotropy parameter $\epsilon$ which is related to the nonlocality parameter $\rho_{G K}$ of the nonlocal London model as $\epsilon=\left(\rho_{G K} / \xi_{0}\right)^{2}\left(1-t^{2}\right) / 12$. To compare with the nonlocal London model results, we take 11] $\lambda(T)=\lambda_{0} / \sqrt{1-t^{2}}$ and $\xi(T)=\xi_{0} / \sqrt{1-t^{2}}$, where $t=T / T_{c}$, so that $\chi=\chi_{0} t / \sqrt{1-t^{2}}$. The dimensionless

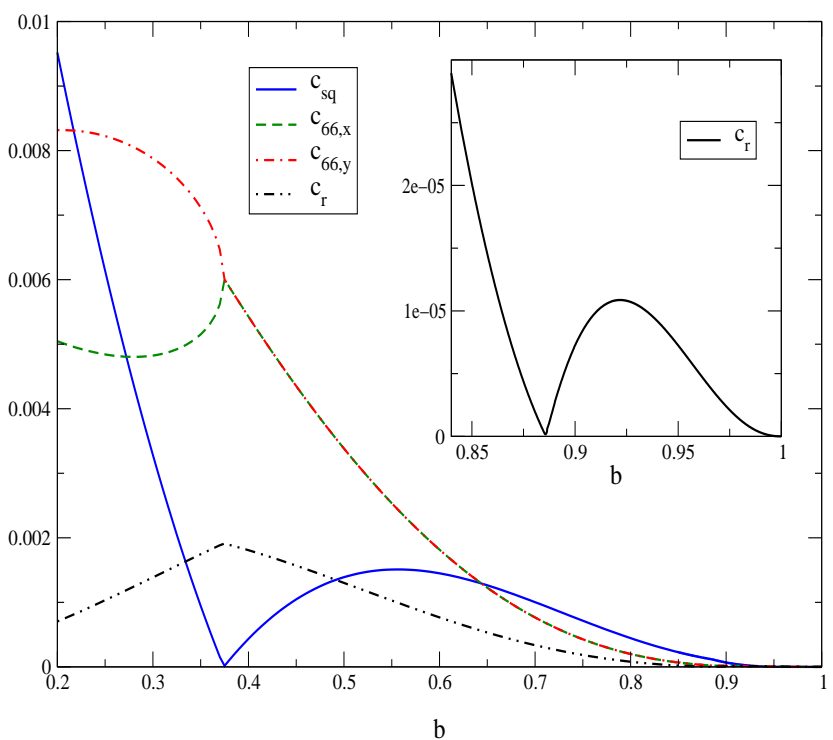

FIG. 1: The elastic moduli versus the reduced field $b$ for $\epsilon=0.11$. In the inset we show the region where the reorientation transition happens, as is signaled by the vanishing of the rotation modulus $c_{r}$.

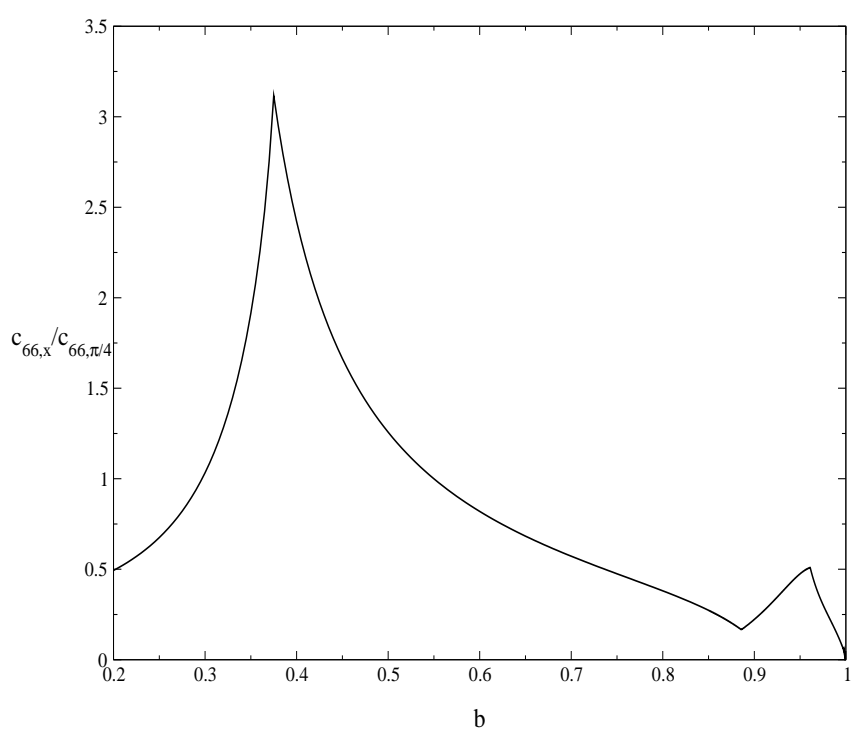

FIG. 2: The ratio $c_{66 x} / c_{66}, \frac{\pi}{4}$ versus the reduced field $b$.

mean squared displacement is then

$$
\overline{u^{2}}=\frac{\chi_{0} \eta}{\sqrt{\left(1-t^{2}\right)}} \frac{b t}{\left[(1-b)^{3} \ln \left(1+\frac{1}{\sqrt{2 b}}\right)\right]^{\frac{1}{2}}},
$$

with $\eta \sim 3$ for $\mathrm{LuNi}_{2} \mathrm{~B}_{2} \mathrm{C}$ [1]. The results of the numerical solution are shown in Fig. 3. for the same parameter values used by Gurevich and Kogan [11], namely, $\rho_{G K}=2.5 \xi_{0}$ and $\chi_{0}=0.0064$. With fluctuations, the phase boundary curves backward and displays the "reentrant" behavior that was also obtained by Gurevich and Kogan. The physical explanation of this feature is simple: thermal fluctuations tend to wash out effects of the 


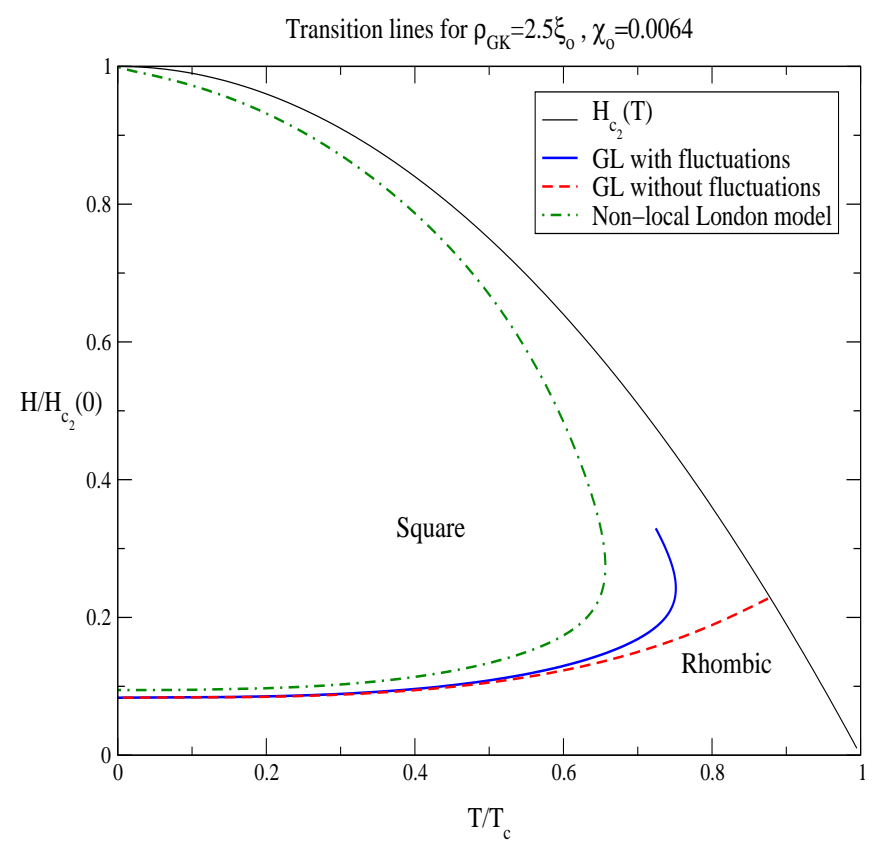

FIG. 3: The phase boundaries in the $H$-T plane separating the square and rhombic vortex lattice obtained from GL theory and the nonlocal London model. The line intercepting $H_{c 2}(t)$ is the mean field GL result.

anisotropy, so that the preferred symmetry of the lattice is rhombic. Note that the abrupt termination of the transition line for the GL theory is an artifact of the perturbative treatment of the anisotropy; at that point the correction term $\Gamma$ ceases to be small and the extended GL theory becomes unstable. While this shortcoming of the model could be remedied by including higher order terms in the GL free energy, the overall qualitative features of the phase diagram are expected to remain unchanged. One such choice would be an additional isotropic term. Nevertheless, the complications introduced in the analysis by the unnecessary extra parameter, would clearly outweigh any potential benefits.

We have used the GL theory with an anisotropic, symmetry breaking term to study the evolution of the vortex lattice and particularly the structural phase transition and its connection with the elastic response of the vortex lattice. We have shown that to obtain the correct phase diagram it is necessary to calculate properties up to second order in the anisotropy; at this order a rotational instability of the square vortex lattice appears. We predict that this instability should be observable in the borocarbides but not in the cuprate superconductors. Including thermal fluctuations in the model we were able to show that the rhombic to square transition line exhibits reentrant behavior, similar to what is obtained within the nonlocal London model, and in qualitative agreement with SANS studies of $\mathrm{LuNi}_{2} \mathrm{~B}_{2} \mathrm{C}$.

We would like to thank V. G. Kogan, A. Gurevich and P. J. Hirschfeld for useful discussions. This work was supported by NSF DMR-9978547.

[1] M. R. Eskildsen et al., Phys. Rev. Lett. 86, 5148 (2001).

[2] M. R. Eskildsen et al., Phys. Rev. Lett. 78, 1968 (1997).

[3] Y. De Wilde et al., Phys. Rev. Lett. 78, 4273 (1997).

[4] H. Sakata et al., Phys. Rev. Lett. 84, 1583 (2000).

[5] L. Ya. Vinnikov et al., Phys. Rev. B 64, 024504 (2001).

[6] R. Gilardi et al., Phys. Rev. Lett. 88, 217003 (2002).

[7] V. G. Kogan et al., Phys. Rev. B 54, 12386 (1996).

[8] V. G. Kogan et al., Phys. Rev. B 55, R8693 (1997).

[9] N. Nakai et al., Phys. Rev. Lett. 89, 237004 (2002).

[10] E. H. Brandt, Rep. Prog. Phys. 58, 1465 (1995).

[11] A. Gurevich and V. G. Kogan, Phys. Rev. Lett. 87, 177009 (2001).

[12] M. Franz, I. Affleck, and M. H. S. Amin, Phys. Rev. Lett. 79, 1555 (1997).

[13] R. Heeb et al., Phys. Rev. B 54, 9385 (1996).

[14] I. Affleck, M. Franz, and M. H. S. Amin, Phys. Rev. B 55, R704 (1997).

[15] M. M. Doria, J. E. Gubernatis, and D. Rainer, Phys. Rev. B 39, 9573 (1989).

[16] U. Klein and B. Pöttinger, Phys. Rev. B 44, 7704 (1991).

[17] A. D. Klironomos and A. T. Dorsey (unpublished).

[18] D. Chang et al., Phys. Rev. B 57, 7955 (1997).

[19] D. Chang et al., Phys. Rev. Lett. 80, 145 (1998).

[20] B. Rosenstein and A. Knigavko, Phys. Rev. Lett. 83, 844 (1999).

[21] K. Park and D. A. Huse, Phys. Rev. B 58, 9427 (1998).

[22] P. Miranović and V. G. Kogan, Phys. Rev. Lett. 87, 137002 (2001).

[23] L. Ya. Vinnikov et al., Phys. Rev. B 64, 220508 (2001). 